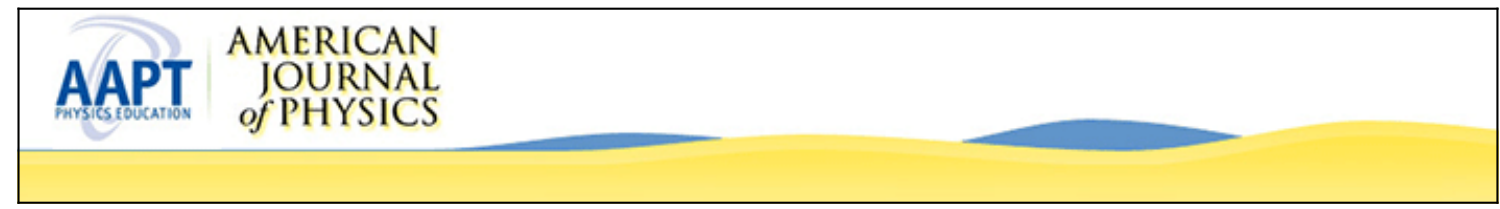

\title{
Undergraduate Experiment on rms Values
}

W. P. Lonc

Citation: American Journal of Physics 39, 957 (1971); doi: 10.1119/1.1986334

View online: http://dx.doi.org/10.1119/1.1986334

View Table of Contents: http://scitation.aip.org/content/aapt/journal/ajp/39/8?ver=pdfcov

Published by the American Association of Physics Teachers

\section{Articles you may be interested in}

Experiment on rms values for undergraduate students

Am. J. Phys. 47, 829 (1979); 10.1119/1.11918

Experiment in Cryogenics for the Undergraduate

Am. J. Phys. 35, 502 (1967); 10.1119/1.1974157

On the Relations between VU-Meter Readings and the rms and Peak Values of Speech

J. Acoust. Soc. Am. 33, 1666 (1961); 10.1121/1.1936662

Advanced Undergraduate Experiments in Physics

Am. J. Phys. 23, 54 (1955); 10.1119/1.1933882

A Simple Device for Finding the RMS Value of Recorded Data

Rev. Sci. Instrum. 23, 243 (1952); 10.1063/1.1746237

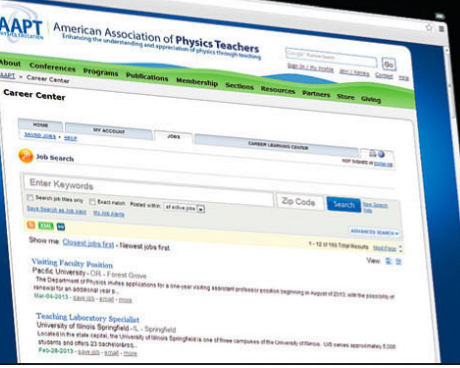

American Association of Physics Teachers

Explore the AAPT Career Center -

access hundreds of physics education and

other STEM teaching jobs at two-year and

four-year colleges and universities.

\section{http://jobs.aapt.org}


functions, $A i(q)$ and $B i(q)$, for large $q$, required in obtaining the classical limit for Eq. (10), are given in Ref. 6.

${ }^{15}$ Discussion of specific experimental considerations and the optimal choice of particles and parameters which can be employed to test the predictions of Eq. (10) are clearly beyond the scope of this Note. Order-of-magnitude criteria for the quantum range of Eq. (10), however, are $a m g \Delta z \cong 10$ and $\hbar^{2} k^{2} / 2 m \cong m g \Delta z$.
${ }^{16}$ For construction of the Green's function in the case of unbounded motion where dispersion does not occur, see I. I. Gol'dman and V. D. Krivchenkov, Ref. 5, pp. 11 and 99. A somewhat complementary question arises with regard to state preparation. It has been argued that it is probably impossible in principle to find two normalizable wave functions associated with particles of different masses which provide identical coordinate and velocity distributions. See Ref. 9.

\section{Undergraduate Experiment on rms Values}

W. P. LONC, S. J.

Physics Department

Saint Mary's University

Halifax, Nova Scotia, Canada

(Received 2 February 1971)

In the study of electric circuits, it seems pedagogically justifiable to perform an experiment which includes both the calibration and experimental verification of the rms value of a waveform, mathematically written as

$$
Y_{\mathrm{rms}}=T^{-1 / 2}\left(\int_{0}^{T} Y^{2}(t) d t\right)^{1 / 2}
$$

Details are presented on the construction, calibration, and utilization of a simple home-made calorimeter consisting of a resistor in thermal contact with a thermistor. The resistor is used to convert the electrical energy into heat, and the thermistor is used as a thermometer. Once the temperature characteristics of the calorimeter have been determined for a known power dissipation in the resistor, subsequent measurements can be made to verify the definition of rms values for any periodic waveform. The calorimeter is adequate for periodic waveforms, inasmuch as the equilibrium temperature is measured; that is, equilibrium between power input, temperature rise, and heat lost to the ambient system.

The calorimeter consists of a $\frac{1}{4}-W$ resistor bonded with epoxy cement to a thermistor. The value of the resistor $(R)$ is chosen so that the available laboratory power supplies and signal generators can supply up to $\frac{1}{4} \mathrm{~W}$ without excessive (say, several percent) waveform distortion. In our experiment, sinusoidal and square waves were to be obtained from an Eico model 377, sine-square generator (about $1 \mathrm{k}$ output impedance) and a Hewlett-Packard model 427, (about 500- $\Omega$ output impedance). Both generators can develop about $5 \mathrm{~V}$ rms across $1 \mathrm{k}$. A simple calculation suggests a value for $R$ of about $100 \Omega$ (it is found that the loading does not lead to excessive waveform distortion).

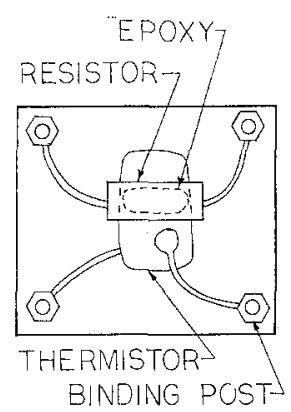

Fia. 1. General layout of the home-made calorimeter. The insulating base is approximately $2 \times 2$ in.

The thermistor was an ordinary disk type unit available from any local electronic supply store for about $\$ 1.00 .^{1}$ The disk shape is recommended because its dimensions can be easily reduced by judiciously applying pressure with ordinary diagonal cutters in such a way as to crack pieces away from the central region (where the thermistor leads are bonded by the manufacturer). Once the maximum amount of thermistor material has been cut away, the rough edges may then be smoothened on an ordinary grinding wheel. The thermistor now has a sufficiently small timeconstant and thermal mass, and a resistance in the order of $1 \mathrm{k} \Omega$. Of course, thermistors designed specifically for temperature measurement would be better, but they are more expensive and more difficult to procure easily.

Bonding is achieved simply and adequately by mixing enough epoxy so that there will be a large-area thermal contact between the resistor $R$ and the thermistor. ${ }^{2}$ To avoid electrical contact between the two leave enough epoxy between the two units to form an insulating layer. Curing time of the epoxy may be shortened by heating near an incandescent lamp.

Once the epoxy has cured, the entire unit is placed on a piece of insulating material (wood, plastic, circuit board, etc.) on which four binding posts have been installed. The wire leads from the resistors are then soldered to the binding posts as in Fig. 1. 


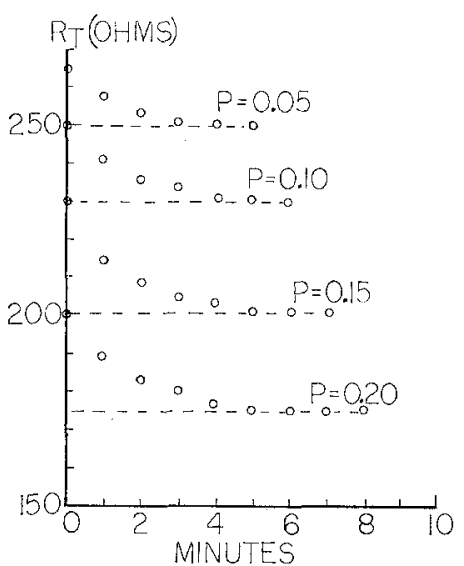

FIG. 2. Typical results for behavior of thermistor resistance with time for various levels of power input to the calorimeter. The broken lines indicate equilibrium values at each power level. $P$ is in units of watts.

On the basis of the definition of rms value, a dc current is a logical waveform for calibration purposes. For a particular value of $R$, the de voltage supply is adjusted to yield power dissipations in $50-\mathrm{mW}$ steps. At the first level $(50 \mathrm{~mW}), R_{T}$ is measured, and then the dc power supply connected to $R$. The de voltage is measured on a high-quality voltmeter if available, and the resulting deflection on the scope is noted, thus calibrating the oscilloscope at the same time. The value of $R_{T}$ is then measured every minute, and each reading is immediately plotted on the graph, as in Fig. 2. After 4 or $5 \mathrm{~min}$, the value of $R_{T}$ should be sufficiently close to equilibrium to decide on its equilibrium value and to proceed to the next power level. The equilibrium value of $R_{T}$ at each power level is plotted, until the graph in Fig. 3 is obtained. This completes the calibration of the calorimeter. Since the oscilloscope has also been calibrated, it now becomes the instantaneousreading voltmeter for the subsequent waveforms for which the de voltmeter is unsuitable.

Three types of waveforms were used: sinusoidal, square, and pulse. Taking into account the available terminal voltage of each generator, one or two values of peak voltage were chosen and the expected power calculated from Eq. (1). The power level should be such as to fall within the limits of the calibration curve (Fig. 3). The waveform should be monitored across $R$, using the calibrated oscilloscope, and the peak value $V_{m}$ is measured. In the absence of generators, the sinusoidal part of the experiment, at least, may be done using the domestic frequency and a suitable isolating step-down transformer.

Since the sinusoidal waveform can be described as $f(t)=V_{m}$ sinct, substitution in Eq. (1) gives

$$
V_{\mathrm{rms}}=(2 \pi)^{-1 / 2}\left(\int_{0}^{2 \pi} V_{m}^{2} \sin ^{2} \omega t d \omega t\right)^{1 / 2},
$$

and then

$$
P=V_{\mathrm{eff}}^{2} / R=V_{\mathrm{rms}^{2}}^{2} / R \text {. }
$$

In a typical experiment, $V_{\mathrm{rms}}$ was chosen to dissipate from 0.05 to $0.2 \mathrm{~W}$ in $R$. The resulting temperature rises of the calorimeter are seen to lie fairly well on the calibration curve (Fig. 3 ).

The appropriate function for a square wave generated by an ac coupled unit such as the Eico is:

$$
\begin{aligned}
V(t) & =V_{m}, & & 0<t<\pi / \omega \\
& =-V_{m}, & & \pi / \omega<t<2 \pi / \omega .
\end{aligned}
$$

Again, a straightforward substitution into the defining

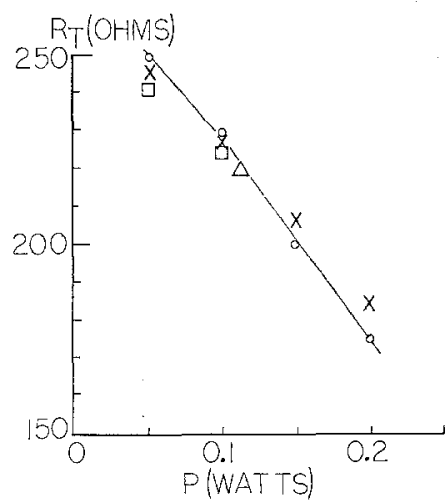

FIG. 3. Calibration curve and data for several waveforms. Symbols: $O$ de calibration points; $\times 60 \mathrm{~Hz}$ sinusoidal; $\square 60 \mathrm{~Hz}$ square wave; $\triangle$ rectangular pulse.

relationship yields the result $V_{\mathrm{rms}}=V_{m}$. If the generator is do coupled and supplies a square wave clamped at zero volts, then the appropriate function is

$$
\begin{aligned}
V(t) & =V_{m}, & & 0<t<\pi / \omega \\
& =0, & & \pi / \omega<t<2 \pi / \omega .
\end{aligned}
$$

In this case,

$$
V_{\mathrm{rms}}=0.707 V_{m}
$$

Hence, the advantage of using a dc coupled oscilloscope is evident. The data for the square wave is shown plotted in Fig. 3, and again the results are convincing.

The pulse generator was adjusted for a width of $50 \mathrm{msec}$ and a period of $200 \mathrm{msec}$. The appropriate function is

$$
\begin{aligned}
V(t) & =V_{m}, & & 0<t<50 \mathrm{msec} \\
& =0, & & 50<t<200 \mathrm{msec},
\end{aligned}
$$

yielding

$$
V_{\mathrm{rms}}=0.5 V_{m} \text {. }
$$


The experiment can be performed in the course of one 3-h laboratory period (calorimeters constructed beforehand) and requires no special precautions to yield satisfactory results. In fact, the data used in this paper are taken from a typical run and are not the average of a larger number of runs.
${ }^{1} \mathrm{GC}$ Electronics \#25-2178, or any thermistor which has a convenient resistance value at room temperature. The convenience depends on the available Wheatstone bridge.

${ }^{2}$ For example, Lepage's Epoxy Glue, size \#350.

\section{An Optimum Lens Configuration for Optical Spatial Filtering}

\author{
B. J. PERNICK \\ Research Department \\ Grumman Aerospace Corporation \\ Bethpage, New York $11 \% 14$
}

(Received 19 November 1970; revised 19 March 1971)

In coherent optical spatial filtering experiments the two-dimensional Fourier transform of an input object scene is modified by selectively attenuating or blocking portions of the light distribution in the back focal plane of a transform lens. Spatial filters alter the transform spectrum by introducing predetermined multiplicative amplitude and/or phase changes in the back-focal-plane light distribution. Amplitude modifications are realized with variable density filters and phase changes with variable thickness or variable refractive index materials.

The light distribution in the back focal plane of the transform lens is a representation of the two-dimensional Fourier transform of an input function, provided the input object is located one focal length ahead of the transform lens. In this conventional optical system a second lens is then used to reimage the desired, modified object scene. ${ }^{1}$ Other constraints require that diffracted rays subtend sufficiently small angles with respect to the optic axis and that these rays are not vignetted by the transform lens aperture. Furthermore, the lens itself should introduce no significant distortions in the diffraction spectrum. If the object is located at a position other than at the front focal plane, the light amplitude distribution in the back focal plane of the transform lens differs from the two-dimensional Fourier transform distribution by just a multiplicative quadratic phase factor. ${ }^{2}$

We are interested here in optical spatial filtering applications that utilize amplitude modification techniques only. By suitable disposition of amplitude spatial filters, the spatial frequency content of the light distribution forming an image can be modulated. In this context, phase variations in the filter plane can be neglected. In particular, the quadratic phase change introduced by arbitrary location of the input object plane is not of immediate concern.

The intent of this note is to provide direction in erecting a modified optical system for spatial filtering experiments. Current systems use three lenses for filtering and reimaging. The modification consists of

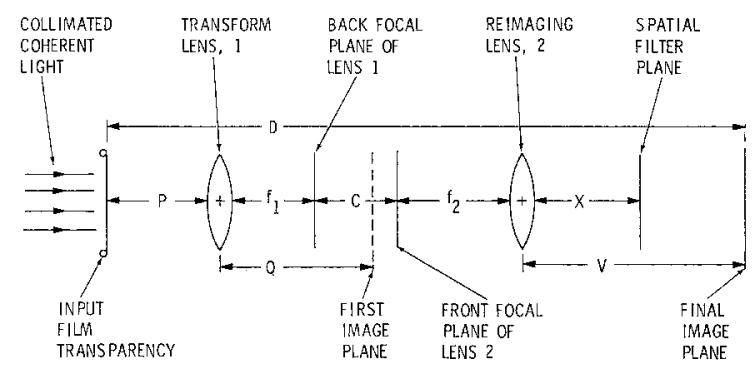

FIG. 1. Two-lens spatial filtering and imaging system.

using a two-lens system to magnify the Fourier transform distribution generated by the first transform lens, as well as to reimage the filtered object scene. Furthermore, it satisfies the requirement that the total length of a two-lens system be a minimum as a practical limitation. The transverse distance in the back focal plane of the transform lens over which a spatial frequency spectrum is contained is limited by the focal length. Thus, within practical limitations on available large focal length lenses, one is faced with a distinet disadvantage in making spatial filters when low spatial frequencies are of interest, or if fine details are to be incorporated into the filter design. A modified two-lens system could be used to provide variable magnification of the frequency spectrum in a filter plane and subsequent reimaging; such a system is shown in Fig. 1.

Let $f_{1}$ represent the focal length of the transform lens, $P$ the object distance, and $Q$ the (first) image distance. The final image is located a distance $V$ from the second lens of focal length $f_{2}$ and is magnified by an amount $M$ with respect to the input object. The length of the optical system from input object to final image is $D$. The second lens is also used to magnify the back focal plane light distribution of the transform lens. The enlarged spatial frequency distribution is at a distance $X$ from the enlarging lens. It is magnified by an amount $m$ with respect to the light distribution in the back focal plane of the transform lens. Rear and front focal planes of both lenses are separated by a distance $C$, as shown in the figure. The spatial frequency distribution to be enlarged is at a position $C+f_{2}$ from the second lens, as illustrated. Since a real, magnified image of this spectrum is required at a distance $X$, it can be shown from the lens equation that the quantity $C$ must satisfy $C+f_{2}>f_{2}$. Consequently, $C>0$. Also, the transform magnification is $m=-X /\left(C+f_{2}\right)$. (Note that $m<0$ to achieve a real, magnified transform 